\title{
Liver damage due to perhexiline maleate
}

\author{
G. B. FORBES, M. O. RAKE, AND D. J. E. TAYLOR \\ From the Departments of Pathology and Medicine, Kent and Canterbury Hospital, Canterbury, \\ Kent, CT1 3NG, UK
}

SUMmaRY Two middle-aged men, who had received perhexiline in recommended dosage, showed clinical and histological evidence of severe hepatic damage, and one of them died. Histological study of the livers showed a striking resemblance to alcoholic hepatitis.

Perhexiline maleate (Pexid) has been used as an antianginal agent for nearly 10 years, and, until recently, there have been few reports of adverse side-effects which could give cause for serious alarm. This communication draws attention to the potential hepatotoxic effect of the drug, a complication that has been recorded by other authors during the past three years (Pelletier et al., 1976; Beaugrand et al., 1977; McDonald, 1977; Bonnet et al., 1978; Pessayre et al., 1979; Lewis et al., 1979).

\section{Case reports}

CASE 1

A white man aged 63 , a teetotaller, presented with a short history of chest pain on exertion, and an electrocardiogram showed ischaemic changes. As treatment with $\beta$ blockade produced unacceptable bradycardia, he was given perhexiline, $100 \mathrm{mg}$ twice a day, together with a small dose of oxyprenolol. He remained well for 10 months on this treatment but then began to feel generally unwell and easily fatigued; he reported loss of balance and was noted to be ataxic. Examination revealed an enlarged liver, and the results of laboratory tests were: serum glutamate oxaloacetate transaminase (SGOT) $139 \mathrm{IU} / 1$ (normal 0-30 IU/1), alkaline phosphatase $112 \mathrm{IU} / 1$ (normal 25-100 IU/l), bilirubin $13 \mu \mathrm{mol} / 1$ (normal 3-17 $\mu \mathrm{mol} / 1$ ), albumin $33 \mathrm{~g} / 1$ (normal 35-50 $\mathrm{g} / \mathrm{l})$, normal electrophoretic protein analysis and negative hepatitis associated antigen ( $\mathrm{HBsAg})$. Serial liver function tests during the next few weeks showed progressively rising SGOT, alkaline phosphatase, and bilirubin levels, and a liver scan suggested parenchymal disease.

The perhexiline was stopped after 13 months' continuous therapy. Later he developed pneumonia

Received for publication 30 May 1979 and signs of hepatic failure; his condition deteriorated despite antibiotic and antihepatic failure therapy and he died three weeks after admission.

\section{Histopathology}

A needle biopsy specimen of liver was taken immediately after death, and histological examination showed a severe degree of liver damage due to focal liver-cell necrosis and active hepatitis. There was a reduction of parenchymal tissue due to wide active septa and micronodular cirrhosis (Fig. 1). The portal tracts and septa showed fibrosis and contained a heavy inflammatory infiltrate which included many neutrophils and no eosinophils. The cytoplasm of most of the liver cells showed degenerative changes which ranged from ballooning to hyaline necrosis with clumps and globules of Mallory's hyaline (Fig. 2). There was evidence of attempted regeneration in the form of atypical hepatocytes with double vesicular nuclei and prominent nucleoli, a feature noted by Lewis et al. (1979). Fatty infiltration was absent, but in other respects the histological picture bore a close resemblance to severe alcoholic hepatitis.

Necropsy was carried out 10 hours after death. The heart was enlarged $(440 \mathrm{~g})$ due to left ventricular hypertrophy, and the coronary arteries showed atherosclerosis with luminal stenosis; there was no evidence of myocardial infarction. The liver was moderately enlarged $(1950 \mathrm{~g})$ and of a dull greenish colour. There was fine micronodular cirrhosis, and there were foci of liquefactive necrosis in parts of the transected organ. The gall bladder and extrahepatic biliary tract were normal, and there was no evidence of neoplasia or cholelithiasis. Histological examination of the liver showed features similar to those seen in the needle biopsy but necrosis was much more advanced and confluent, and this was attributed to superimposed postmortem autolysis. 


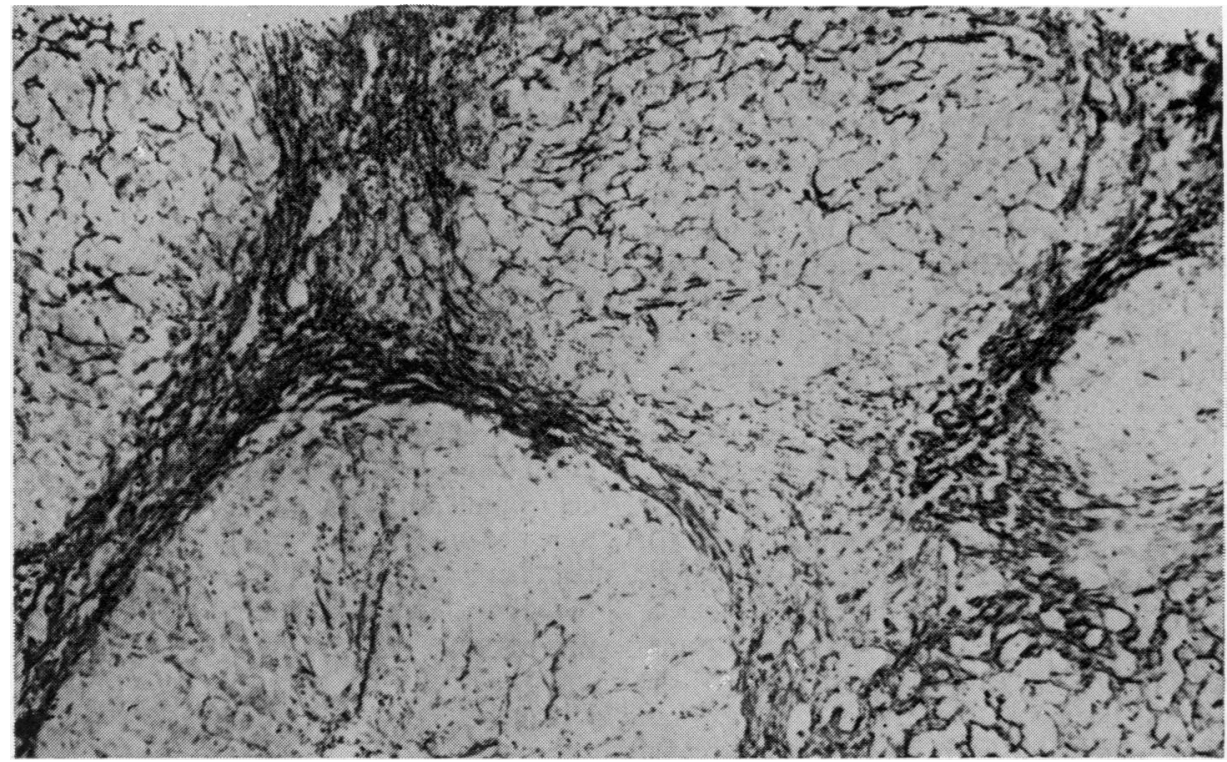

Fig. 1 Case 1. Micronodular cirrhosis. Liver biopsy. Reticulin $\times 100$

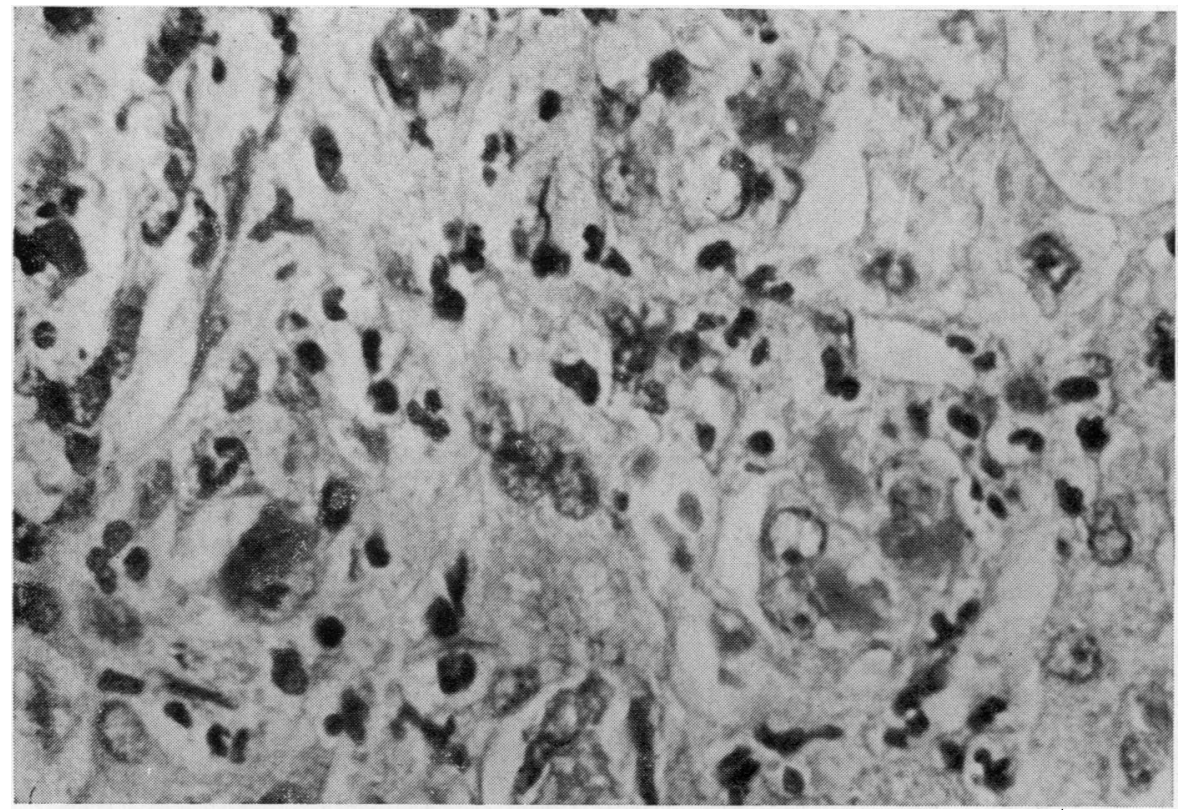

Fig. 2 Case 1. Inclusions of Mallory's hyaline, neutrophil infiltration, and nuclear atypia. Liver biopsy. Haematoxylin and eosin $\times 800$ 


\section{CASE 2}

A 63-year-old white man had complained of angina pectoris for several years, and during the 14 months before admission he had received perhexiline in a dose of $100 \mathrm{mg}$ twice a day. He also suffered from asbestosis and recurrent attacks of chronic bronchitis, and for three months before admission he had complained of general ill health, anorexia, weight loss, and jaundice. He admitted to having consumed half a bottle of spirits daily in the past but his alcohol consumption had been much reduced during recent months. Examination showed an enlarged liver, and an isotopic liver scan showed generalised diminished uptake; a needle biopsy specimen was taken. Biochemical tests gave the following results: SGOT 227 IU/1 (normal 0-30 IU/1), alkaline phosphatase $560 \mathrm{IU} / 1$ (normal 25-100 IU/l), bilirubin $54 \mu \mathrm{mol} / 1$ (normal 3-17 $\mu \mathrm{mol} / \mathrm{l}$ ), 5 nucleotidase $60 \mathrm{IU} / 1$ (normal 0-17 IU/1), $\gamma$ glutamyl transpeptidase ( $\gamma$ GT) $1160 \mathrm{IU} / 1$ (normal 10-55 IU/1); the HBsAg test was negative.

Perhexiline was withdrawn and he was treated with prednisolone, $20 \mathrm{mg}$ twice daily. On this regime he remains fairly well two months later; his liver is still enlarged and there has been only slight im- provement in liver function tests: SGOT $46 \mathrm{IU} / 1$, alkaline phosphatase $480 \mathrm{IU} / \mathrm{l}$, bilirubin $14 \mu \mathrm{mol} / \mathrm{l}$, and $\gamma$ GT 945 IU $/ 1$.

\section{Histopathology}

Sections of a needle biopsy specimen of liver (Fig. 3) showed a histological picture that was almost identical with that seen in the needle biopsy of case 1 , and it was this striking similarity that drew our attention to the possibility of a common aetiological factor. The changes were perhaps less pronounced in this nonfatal case, but the dominant features were again severe liver-cell damage with deposition of Mallory's hyaline in some of the degenerate cells, aggregates of neutrophils, and binucleate hepatocytes. The septa were oedematous and contained an excess of collagen tut there was no obvious evidence of cirrhosis in reticulin-stained preparations. Fatty infiltration was minimal.

\section{Discussion}

That perhexiline could have a deleterious effect on liver function has been suspected for some time, and several separate clinical trials have shown that

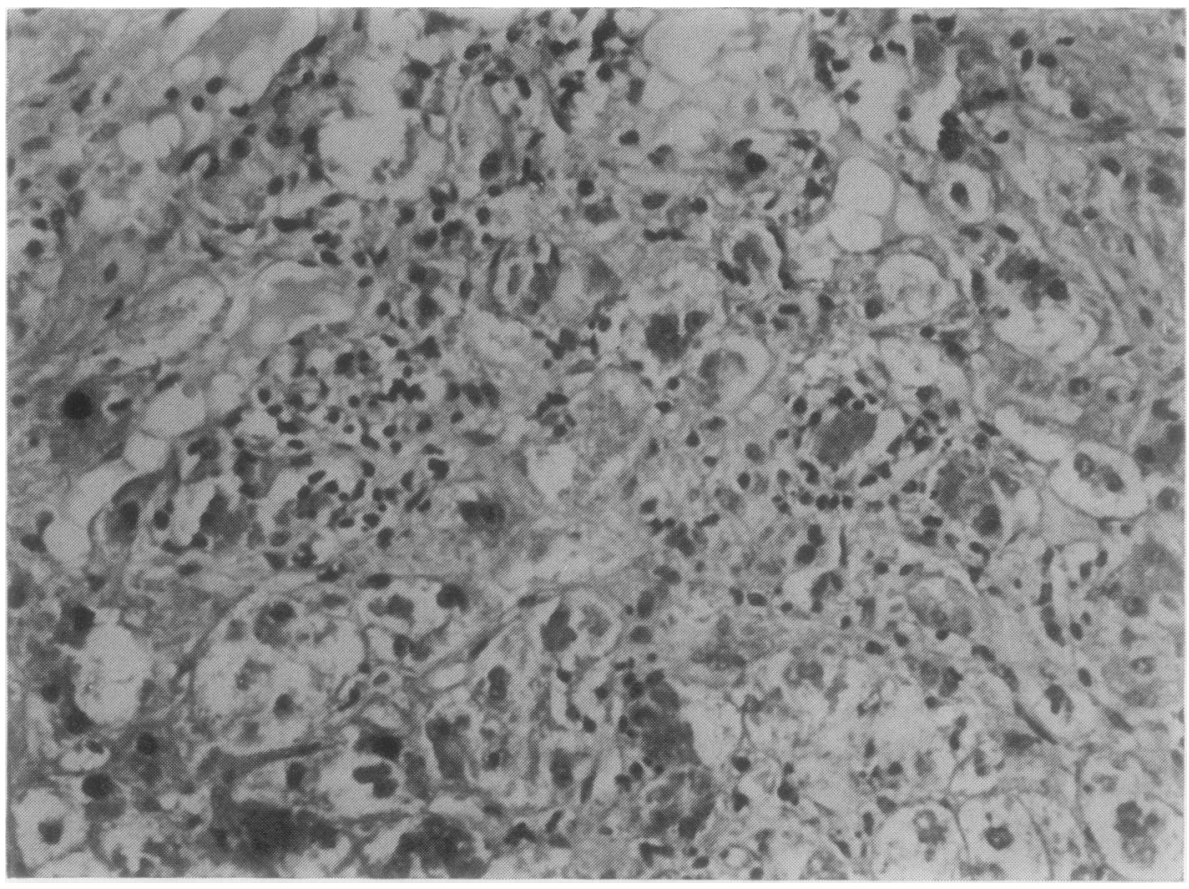

Fig. 3 Case 2. Balloon degeneration of hepatocytes, Mallory's hyaline, and inflammatory infiltrate. Liver biopsy. $H$ and $E \times 300$ 
approximately $10 \%$ of patients receiving the drug have raised liver enzyme levels. The effect was regarded by Howard and Russell Rees (1976) and Lockhart and Masheter (1976) as relatively mild and reversible, but they advised treating at the smallest effective dose or withdrawing the drug altogether if abnormal liver function tests persisted. However, recent reports, especially from France where perhexiline is prescribed frequently, together with the two cases reported herein, indicate that this form of therapy may be responsible occasionally for a much more severe degree of hepatic damage which may lead to death from liver failure. Beaugrand et al. (1977) reported two cases, one of which terminated fatally. The clinical and histological features described in their report parallel closely those encountered in our cases; in particular, they observed in sections of the liver micronodular cirrhosis, zones of hepatocellular nezrosis, and numerous Mallory bodies. Similar morphological features were seen in a fatal case reported by Bonnet et al. (1978). In a recent paper, Pessayre et al. (1979) have reviewed the relevant literature and recorded two fatal cases of cirrhosis after prolonged administration of perhexiline. The same pattern of histological changes was seen in non-fatal cases reported by McDonald (1977, 4 cases) and by Lewis et al. (1979, 2 cases). All patients had received perhexiline for long periods, ranging from 9 to 36 months.

Our two cases confirm the observation that there is an impressive resemblance between the histological picture seen in perhexiline-treated patients and that seen in alcoholic hepatitis and cirrhosis. However, there appears to be no significant association between alcohol consumption and perhexiline-induced hepatitis. The four fatal cases reported from France were abstainers, as were the two patients of Lewis et al. and three of McDonald's patients, although the latter's fourth patient admitted to a large daily intake of alcohol. One of our patients had been a moderately heavy drinker in the past but had reduced his alcohol intake appreciably in recent months and consumed no alcohol during his stay of several weeks in hospital; the other was a teetotaller. Their treatment did not include any other drug that is known to have potential hepatotoxicity, and the overall evidence suggests strongly that the liver damage was directly attributable to perhexiline.

Apart from alcoholic hepatitis there are few diseases of the liver in which Mallory's bodies are readily recognised. The list includes conditions that are unlikely to be confused with hepatitis due to perhexiline, for example, Wilson's disease, Indian childhood cirrhosis, small-bowel bypass operations, and chronic cholestasis. Mallory's bodies can also be found in liver biopsies from patients with diabetes and obesity, and these commoner diseases could present diagnostic problems as they may mimic alcoholic hepatitis closely.

Evidence is now accumulating which incriminates perhexiline treatment as a possible cause of severe hepatitis with or without cirrhosis, and the pathologist should be aware of this possibility when confronted with a histological picture resembling alcoholic hepatitis. Clinicians for their part should ensure that full and specific information on drug treatment is made available to the pathologist when submitting liver biopsy specimens.

It is recommended that patients undergoing treatment with perhexiline should have careful periodic monitoring of liver function, including liver biopsy, and the drug should be withdrawn at the first sign of dysfunction.

We thank Mr E. W. Spice, FIMLS, for the photomicrographs.

\section{References}

Beaugrand, M., Chousterman, M., Callard, P., Camilleri, J. P., Petite, J. P., and Ferrier, J. P. (1977). Hépatites au maléate de perhexiline (PEXID ${ }^{\mathrm{R}}$ ) évoluant vers la cirrhose malgré l'arrêt du traitement ( 2 cas). Gastroentérologie Clinique et Biologique, 1, 745-750.

Bonnet, P., Jourdan, J., Janbon, F., Michel, H., and Bertrand, A. (1978). Cirrhose hépatique après deux ans de traitement par le maléate de perhexiline (Letter). Nouvelle Presse Médicale, 7, 208.

Howard, D. J., and Russell Rees, J. (1976). Long-term perhexiline maleate and liver function. British Medical Journal, 1, 133.

Lewis, D., Wainwright, H. C., Kew, M. C., Zwi, S., and Isaacson, C. (1979). Liver damage associated with perhexiline maleate. Gut, 20, 186-189.

Lockhart, J. D. F., and Masheter, H. C. (1976). Report of a multi-centre monitored release study of perhexiline maleate in the prevention of angina pectoris. British Journal of Clinical Practice, 30, 172-177.

McDonald, G. S. A. (1977). Liver damage after perhexiline maleate (Letter). Lancet, 1, 1056.

Pelletier, M., Lambert, R., Paliard, P., and Bory, R. (1976). Hépatite toxique subaiguë après traitement par maléate de perhexiline (Letter). Nouvelle Presse Médicale, 5, 1070.

Pessayre, D., Bichara, M., Feldmann, G., Degott, C., Potet, F., and Benhamou, J. P. (1979). Perhexiline maleate-induced cirrhosis. Gastroenterology, 76, 170177.

Requests for reprints to: Dr G. B. Forbes, Department of Pathology, Kent and Canterbury Hospital, Canterbury CT1 3NG, UK. 